\title{
Advanced Analysis of HVDC Electrodes Interference on Neighboring Pipelines
}

\author{
Yu Gong1, Chunlin Xue2, Zhilei Yuan'2, Yexu Li³, Farid Paul Dawalibi ${ }^{3}$ \\ ${ }^{1}$ China Southern Power Grid, Guangzhou, China \\ ${ }^{2}$ Huadong Power Design Institute, Shanghai, China \\ ${ }^{3}$ Safe Engineering Services \& Technologies Itd., Laval, Quebec, Canada \\ Email: yexu.li@sestech.com, info@seschina.cn
}

Received February 2015

\begin{abstract}
This paper focuses on advanced analysis techniques and design considerations of DC interference generated by HVDC electrodes during normal bipolar and temporary monopolar operations on neighboring metallic utilities, with a special emphasis on buried gas and oil pipelines. This study examines the level of pipeline corrosion, the safety status in the vicinity of exposed appurtenances and the impact of DC interference on the integrity of insulating flanges and impressed current cathodic protection (ICCP) systems. Computation results obtained for different soil models show that different soils can lead to completely different DC interference effects. The results and conclusions presented here can be used as a reference to analyze the severity of DC interference on pipelines due to proximate HVDC electrodes.
\end{abstract}

\section{Keywords}

HVDC Electrode, DC Interference, Pipe-to-Soil Potential, Polarization Potential, Insulating Joint (Flanges), Rectifier, Touch Voltage, Corrosion, Safety, Impressed Current Cathodic Protection

\section{Introduction}

HVDC networks have been widely implemented in China in recent years. HVDC has proved to be well suited to specific applications, including long-distance power transmission, relatively long cable interconnections, interconnections between large isolated HVAC systems, and asynchronous tie-lines between HVAC systems. During normal bipolar operations and particularly during temporary monopolar operations of the HVDC lines, DC currents injected into the soil result in an electric field that can introduce excessive currents and voltages in nearby metallic utilities, such as pipelines. Consequently, such current can cause or accelerate DC corrosion, damage rectifiers and insulating flanges or joints and can threaten the safety of personnel working at valve and test stations as well as along the pipe. Due to the significant increase of HVDC systems, the concerns on their possible adverse impact on the environment have accelerated the need for accurate approaches to analyze HVDC adverse effects on neighboring buried metallic utilities and development of appropriate effective and economical mitigation measures.

This paper discusses recent advances and new developments achieved in the analysis of electromagnetic in- 
terference generated by HVDC ground electrodes on neighboring metallic utilities, with a particular emphasis on buried pipelines. This study is based on a realistic case and has been carried out using a computer model that reproduces accurately the existing installations. The study includes comparisons between field measurements and computed results in order to validate the computation method and computer models that were developed to describe accurately the existing system. It examines the level of pipeline corrosion, the safety status in the vicinity of exposed appurtenances and the impact of DC interference on the integrity of insulating flanges and impressed current cathodic protection (ICCP) systems. A future paper will deal with advanced mitigation measures to reduce the interference level to acceptable values following various design standards and criteria.

\section{HVDC Electrode Interference Effects on Pipelines and Design Standards}

HVDC systems may operate in such a manner that continuous (normal operation) or periodic (monopolar operation) direct current follows an earth path between HVDC system terminals. During such operations, a portion of the DC current that is flowing between the two HVDC electrodes is captured by the pipelines and associated grounding systems located in zones where the earth potentials are high and are discharged back to soil at locations where the earth potentials are lower. More precisely, a pipeline may collect, conduct, or discharge a portion of this current depending on the location of the pipeline and the polarity of the HVDC ground electrode. When the electrode near the pipeline is operating in positive mode, the current from the electrode is collected by the pipeline in the region located in the vicinity of the electrode. The current is then discharged from the pipeline at locations remote from the electrode; resulting in corrosion at these points. When the HVDC electrode near the pipeline is operating in negative mode, the current is discharged from the pipeline in the vicinity of the electrode and collected in the area far from the electrode. In this case, corrosion occurs near the electrode. In addition, coating damage due to disbonding can also occur if the pipeline is polarized negatively beyond a safe level as a result of excessive current collection. Figure 1 illustrates this DC interference mechanism due to the operation of an HVDC electrode.

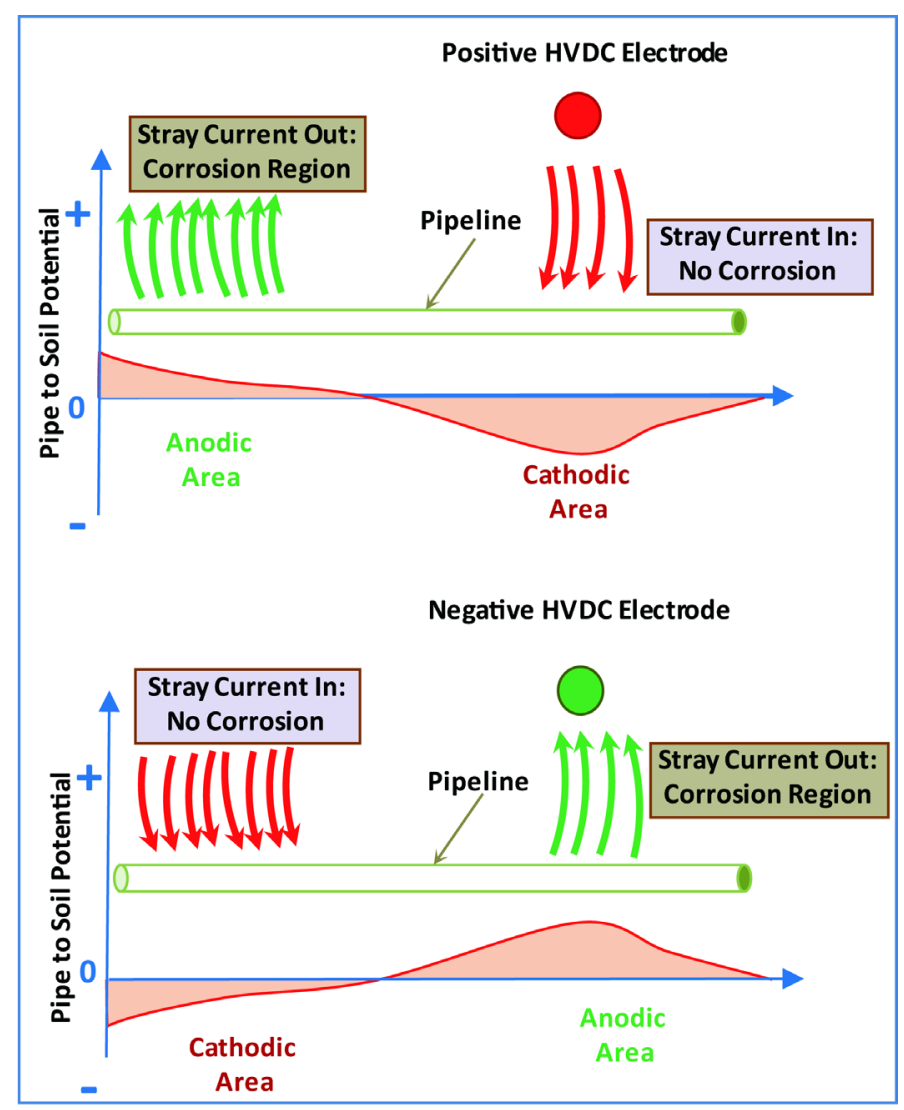

Figure 1. Mechanism of HVDC electrode interference. 
The European Committee for Electrotechnical Standardization BS/EN50162-2004 [1] and Chinese National Standard GB/T 21447-2008 [2] state that: 1) Protective measures must be applied if the pipe leakage current density is more than $1 \mu \mathrm{A} / \mathrm{cm}^{2}$ or the cumulative corrosion amount (thickness) affects the safe operation; 2) DC interference exists if the pipe-to-soil potential (polarization potential) is higher than $20 \mathrm{mV}$ positive shift to the pipe natural potential (galvanic series potential) or the DC soil potential gradient near the pipe is greater than 0.5 $\mathrm{mV} / \mathrm{m}$. When the pipe-to-soil potential is higher than $100 \mathrm{mV}$ positive shift to the pipe natural potential (galvanic series potential), protective measures must be applied; 3) For a new pipeline, if a pipeline route is in the zones where the DC soil potential gradient is greater than $2.5 \mathrm{mV} / \mathrm{m}$, the pipeline may be subject to DC interference and therefore must be evaluated. Protective measures must be applied to mitigate the excessive DC voltages and currents.

The National Association of Corrosion Engineers (NACE) Standard [3] states that the protection criterion for corrosion is a negative (cathodic) voltage of at least $0.85 \mathrm{~V}$ with respect to a saturated copper/copper sulfate electrode. Determination of this voltage is to be made after the polarization has been achieved with the protective current applied. The generally accepted value below which coating damage due to disbonding may become significant is $-1.5 \mathrm{~V}$.

The primary concern of the personnel safety is to satisfy ANSI/IEEE Standard 80 safety criteria at aboveground pipeline appurtenances.

\section{Measured vs. Computed Interference Levels}

The system described in this section will be used as our example for most of the following discussions. The computer model, including a $609.5 \mathrm{~mm}$ diameter pipeline and the three nearby HVDC ground electrodes, that were under different operation modes, is shown in Figure 2.

The studied pipeline covers a distance of over $270 \mathrm{~km}$. The closet point of the pipeline is about $7 \mathrm{~km}$ away from the HVDC electrode operating in monpolar mode. The soil was measured and analyzed and was found to have the four-layer structure as shown in Table 1. The injected current into the monpolar HVDC ground electrode is 3232 A while the unbalanced currents into the soil for the other two electrodes that are under normal operation are $61 \mathrm{~A}$ and $18 \mathrm{~A}$, respectively. The pipeline coating resistance is assumed to be $100,000 \mathrm{ohm}-\mathrm{m}^{2}$.

The pipe-to-soil potentials at various locations along the pipeline were measured. The results of the measured and computer simulation are shown in Figure 3. As it can be seen, the theoretical computing results (the blue curve), compared with the measured results (the orange dots), agree with each other very well. This conductive coupling between the HVDC electrodes and pipeline results in soil currents collected then discharged by the pipeline. The computer model and computation method are validated.

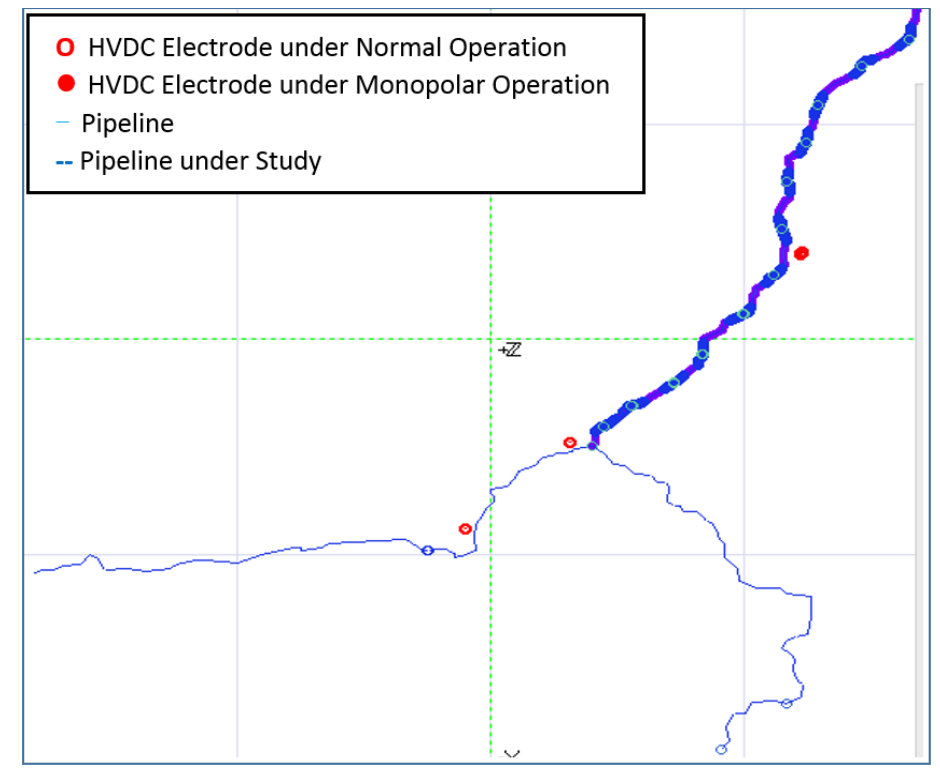

Figure 2. Top view of the studied network. 
Table 1. Existing soil model structure.

\begin{tabular}{cc}
\hline Depth (m) & Resistivity (ohm-m) \\
\hline $0 \sim 2$ & 200 \\
$2 \sim 10$ & 500 \\
$10 \sim 30$ & 200 \\
$30 \sim 300$ & 1000 \\
$>300$ & 3500 \\
\hline
\end{tabular}

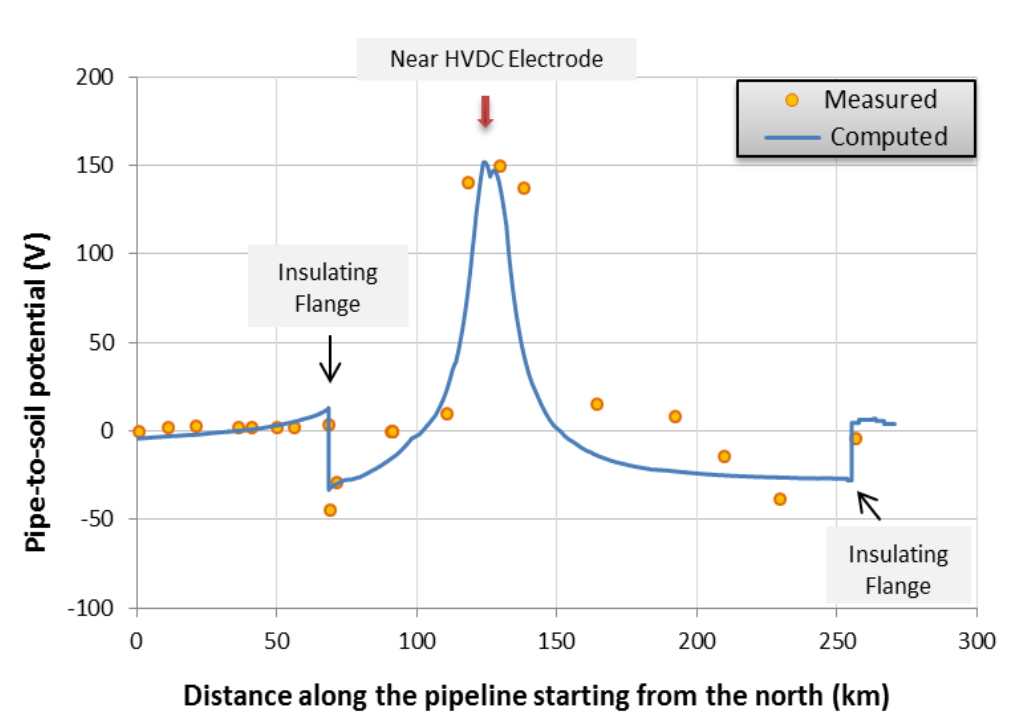

Figure 3. Computed and measured DC interference levels.

\section{Pipeline Corrosion Analysis}

Leakage current density, pipe-to-soil potential, electric field gradient outside the pipe and current density across a $1 \mathrm{~cm}^{2}$ holiday are important quantities when a pipe is assessed for its corrosion activity status. Considering the dc voltage drop caused by the pipeline conductor longitude resistance and knowing that the three electrodes are operating in positive mode, the pipeline status is examined based on the soil structure shown in Table 1. The resulting pipeline leakage current density is displayed Figure 4. This figure shows that when the monopolar operation electrode is positive, some current tends to be collected by the pipeline at points near the electrode. This current is carried away towards both ends of the pipeline, where it is discharged progressively.

The pipe-to-soil potentials and the electric field gradient outside the pipe are plotted in Figure 5 and Figure 6, respectively. Clearly, corrosion occurs seriously along most, if not the entire length of the pipeline, if no mitigation is applied or if the cathodic protection system is not adjusted accordingly. Furthermore, coating disbanding may occur at the south end locations because pipe-to-soil potentials remain below the $-1.5 \mathrm{~V}$ limit. The maximum electric field gradient is about $93.7 \mathrm{mV} / \mathrm{m}$.

The current density in coating defects (referred to as holidays) is one of the key parameters used to evaluate the corrosion rate. The calculation of coating potential from the coating impedance and radial leakage current density, indicates that the holiday current can be estimated fairly accurately along the entire pipeline based on the coating stress voltage, assuming that there are enough grounds along the pipeline length (including its imperfect coating) to establish the external pipeline potential (which is the case in our model). In this case, a small holiday introduces only a small perturbation to the external potential. The holiday current is then estimated as the ratio of the "coating potential difference" to the product of the holiday resistance by the holiday base area. The holiday current through a $1 \mathrm{~cm}^{2}$ defect is shown in Figure 7. The maximum current through such defect is $14.61 \mathrm{~mA}$. Consequently, for a $1 \mathrm{~cm}^{2}$ small defect occurring anywhere along pipeline perforation time may occur in about 39 days! This estimate is based on the knowledge that each ampere of current discharging continuously 


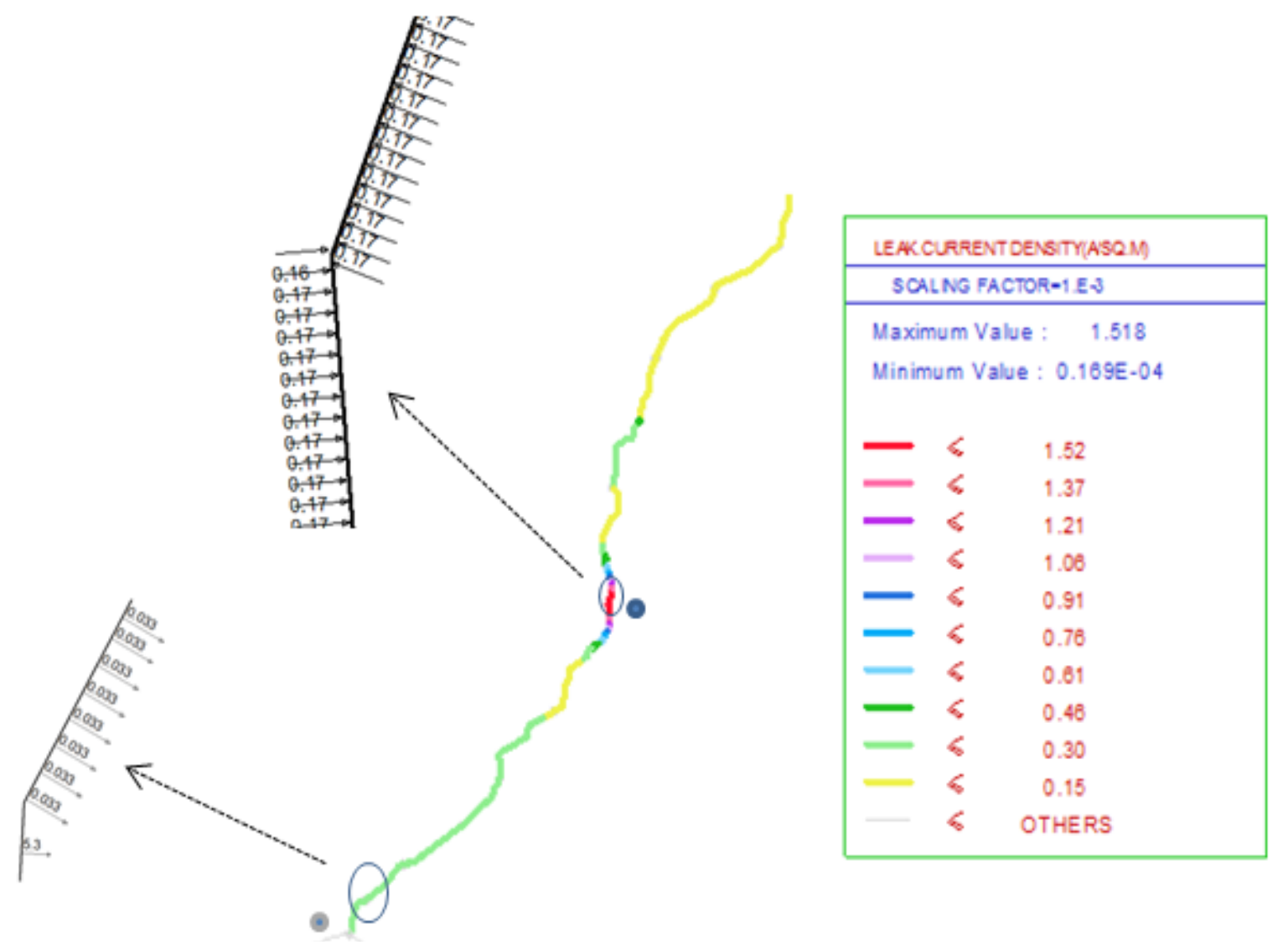

Figure 4. Leakage current along the pipeline.

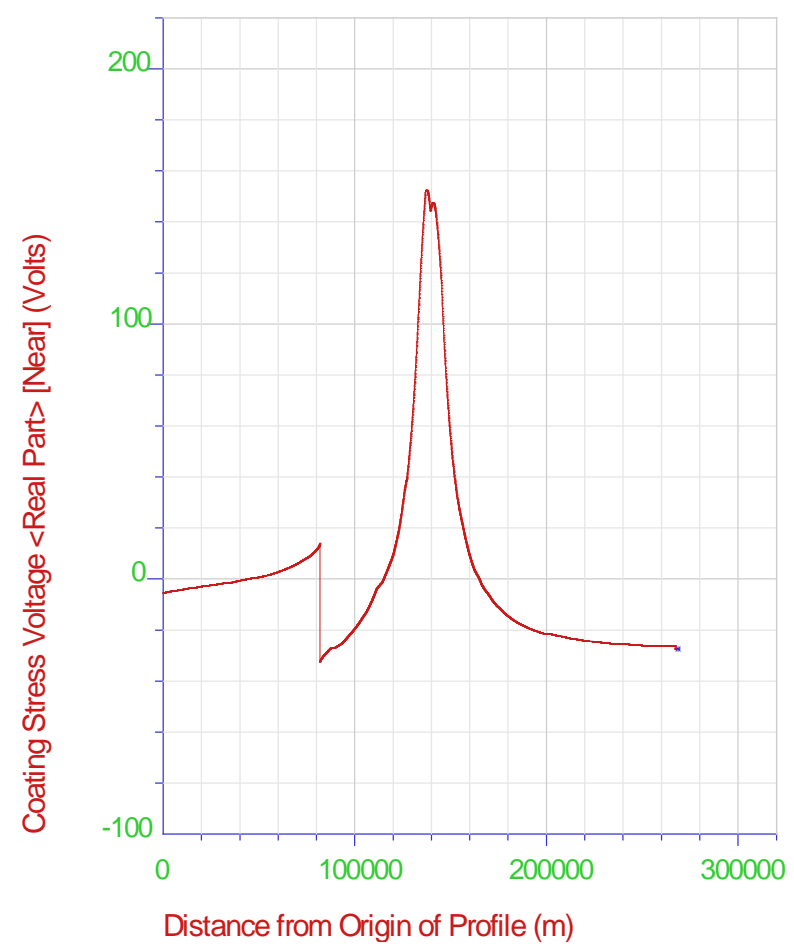

Figure 5. Pipe-to-soil potential. 


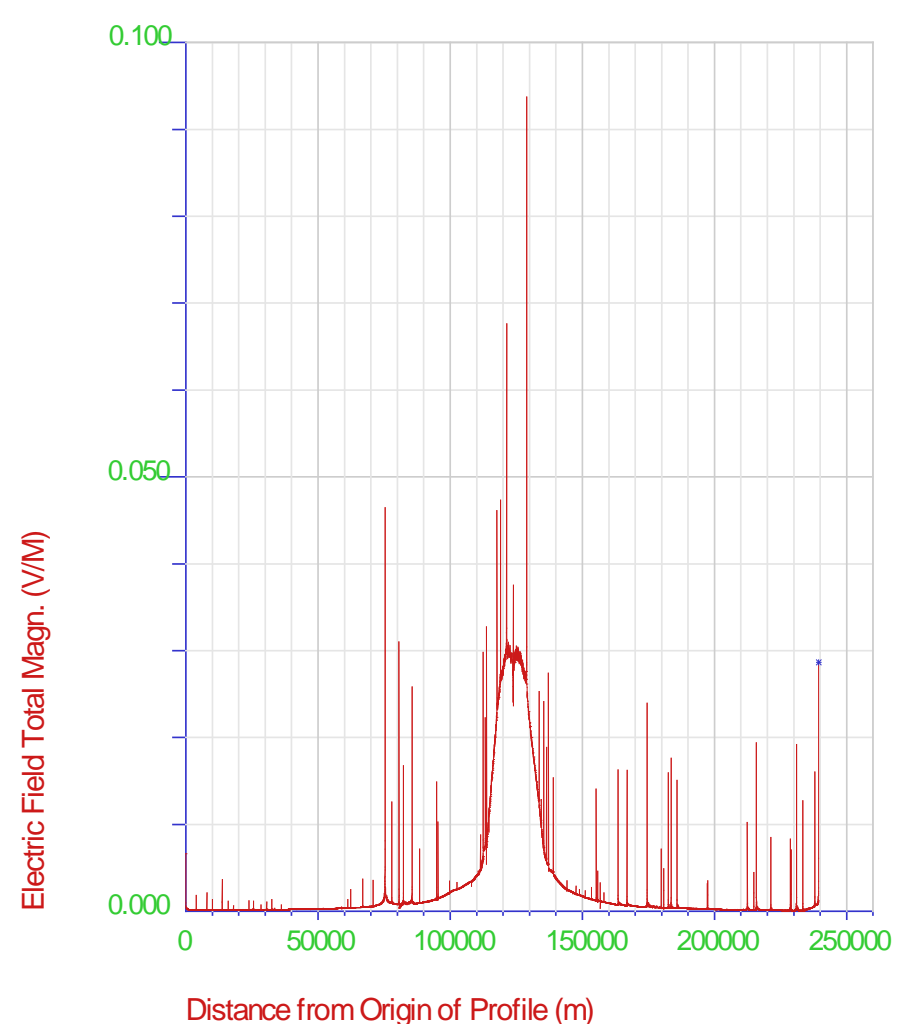

Figure 6. Electric field outside the pipe.

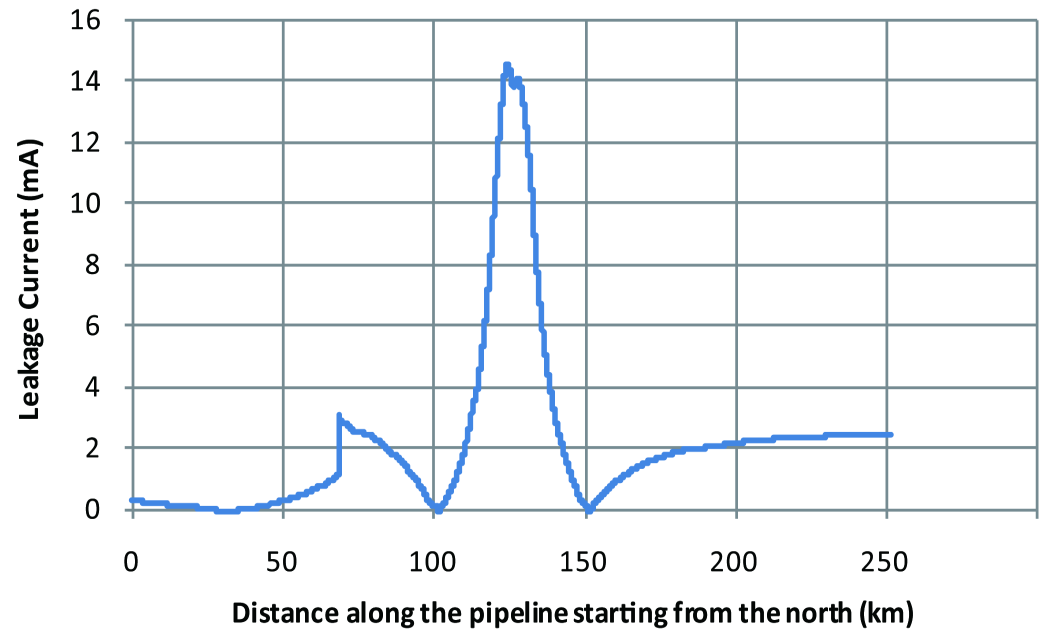

Figure 7. Leakage current along the pipeline through a one (1) $\mathrm{cm}^{2}$ holiday.

from one location on a ferrous structure remove approximately $9.1 \mathrm{~kg}$ of iron in a year's time [4]. Since the pipe wall thickness is $1.84 \mathrm{~cm}$, in the absence of any mitigation measures and cathodic protection system, the pipeline fast perforation time is about 39 days.

Mitigation measures aimed at reducing safety hazards and DC interference levels must be designed if the interference level is high. Presently, there are a few available techniques, such as increasing separation distance between the electrode and the pipeline, adding insulating flanges at appropriate locations, installing lumped concentrated grounds or continuous mitigation wires, etc. Regardless of the method used, however, it is crucial that accurate computer models be developed, taking the soil structure into account. Mitigation techniques and their efficiency will be discussed in a subsequent paper. 


\section{Safety at Valve and Testing Stations}

Touch and step voltages should be evaluated for personnel safety at valve and testing stations. In order to consider all elements associated with the pipeline, a full computer model of the electrode and pipeline network system, including valve grounding grids, insulting flanges, anode beds and rectifiers was built.

Various cases have been analyzed and touch and step voltages for all stations have been examined. However, in this paper, only the results for a typical case are reported to illustrate the HVDC conductive coupling level at valve stations along the pipe.

Figure 8 shows the touch voltages to the pipe, the touch voltages to the conductors of the valve grid aboveground and step voltages at the stations. The maximum computed station touch voltage is $47.9 \mathrm{~V}$ and it occurs at the station that is close to the monopolar HVDC electrode ground. This value was considered below the touch voltage safe design limit required at this restricted access location for this project. The maximum step is only $0.04 \mathrm{~V}$, well below the safe design limit required in this project.

\section{Integrity of Insulating Flanges and ICCP Systems}

To ensure the effectiveness of cathodic protection, rectifier stations are built along pipelines. Furthermore hydraulic valve stations are a very important part of the pipeline system. In case of an emergency, it automatic shuts-off the valve, cuts off the downstream flow of gas or oil to prevent a catastrophic leakage. Insulating joints at a valve station separate the pipeline and its grounding systems. These are essentially electrically isolated from one another in order to prevent unnecessary leakage of the cathodic protection current. However, under HVDC electrode monopolar operations, the transferred voltages across the insulated joints of the pipeline network can be large enough to activate the lightning protectors or breakdown the insulating flanges. Consequently, damage to the integrity of the pipeline may occur under normal operations.

To evaluate the impact of the HVDC electrode operations on the insulating flanges correctly, the voltages across the insulating joints at all stations was carried out. The maximum voltage across the insulating joint in our studied system was about $100 \mathrm{~V}$. The DC impulse breakdown voltage of the insulating joints is on the order of $1000 \mathrm{~V}$. Therefore, all insulating joints are not subject to excessive stress voltages and will remain in good working conditions.

Gas and oil hazardous product pipelines are routinely protected by a coating supplemented with cathodic protection. An ICCP (impressed current cathodic protection) system for a pipeline consists of a DC power source,

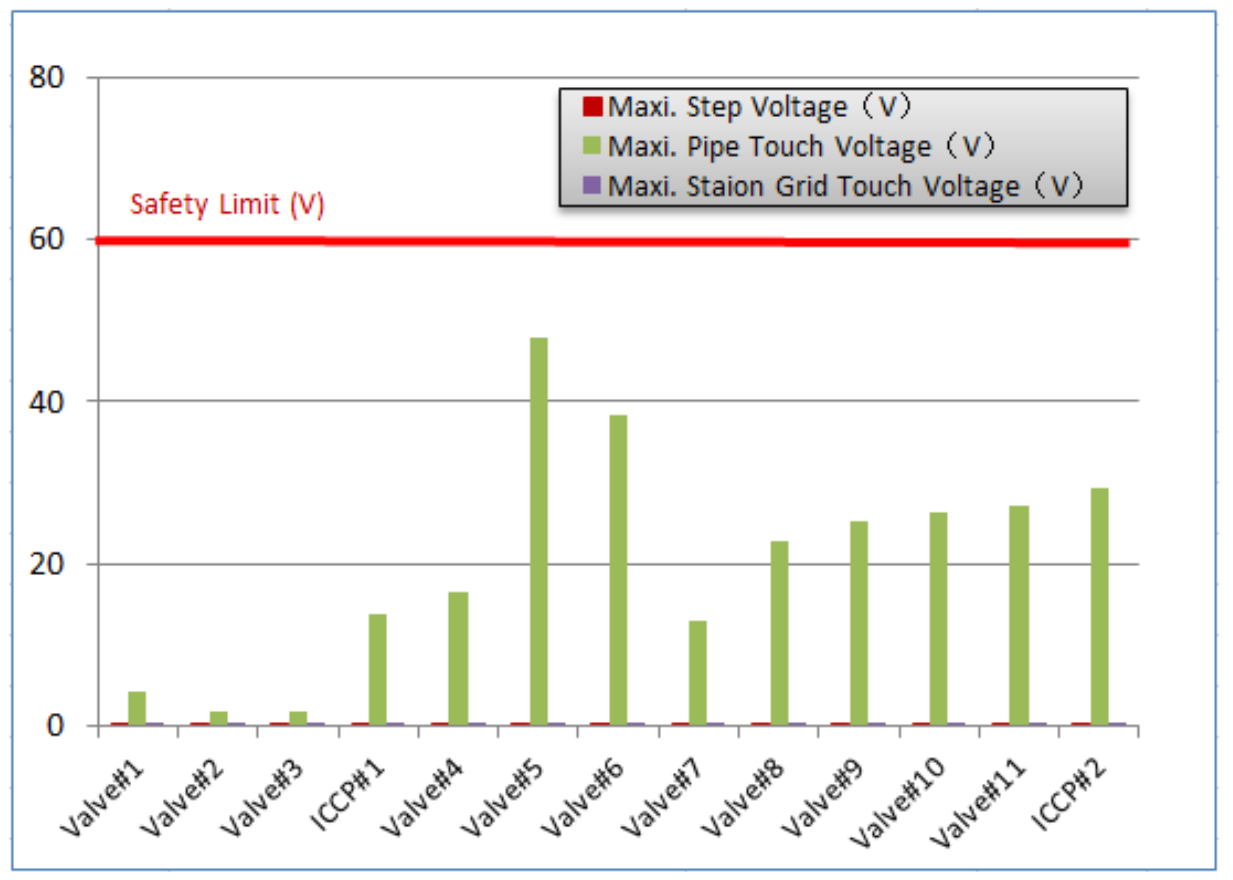

Figure 8. Maximum touch and step voltages at aboveground appurtenances. 
often an AC powered transformer rectifier and an anode, or array of anodes buried in the ground. The DC power source would typically need a constant DC output of a specific current and voltage, depending on several factors, such as the size of the pipeline and coating quality. The positive DC output terminal is connected via cables to the anode array, while another cable connects the negative terminal of the rectifier to the pipeline, preferably through junction boxes to allow measurements to be taken. The rectifier stack is comprised of silicon diodes in series in a bridge circuit. The diodes can be damaged due to switching surges or large steady state DC or AC gradients in the soil during monopolar operation.

There are four ICCPs along the studied pipeline. The systems, including the anode arrays and the cables, are modeled as is and the voltages on the rectifiers are examined. The results are listed in Table 2 . They appear to be all below the rectifier diodes breakdown voltage which is typically about a few hundred volts.

\section{Effects of Soil Structure}

The magnitude of HVDC transferred ground potential rise is strongly influenced by the soil structure. It decreases with increasing distance away from the HVDC electrode, but the rate of decrease depends upon the soil structure. Due to its key importance, this section demonstrates the influence of the soil structure on the predicted DC conductive levels. Three types of soil structures are examined based on the same network shown in Figure 1: A $100 \Omega$-m uniform soil (Soil \#1), a two-layer soil consisting of a $2 \mathrm{~m}$ thick, $10 \Omega$-m top layer overlying a 2000 $\Omega$-m infinitely thick layer (Soil \#2) and a two-layer soil similar to the preceding one except for the layer resistivities that are reversed (Soil \#3). Figure 9 shows the pipe-to-soil potentials due to the transferred potentials through the soil (conductive coupling effects) for the three soil models. Figure $\mathbf{1 0}$ plots the pipe-to-soil potential as a percentage of the GPR of the HVDC electrode that is under monopolar operation.

Table 2. Voltages across rectifiers.

\begin{tabular}{cccc}
\hline Rectifier & Anode GPR (V) & Pipe GPR (V) & Voltage across Rectifier (V) \\
\hline$\# 1$ & 17.2 & 21.5 & $\mathbf{4 . 3}$ \\
$\# 2$ & 121.8 & 72.5 & $\mathbf{4 9 . 3}$ \\
$\# 3$ & 27.8 & 53.3 & $\mathbf{2 5 . 5}$ \\
$\# 4$ & 20.5 & 49.7 & $\mathbf{2 9 . 2}$ \\
\hline
\end{tabular}

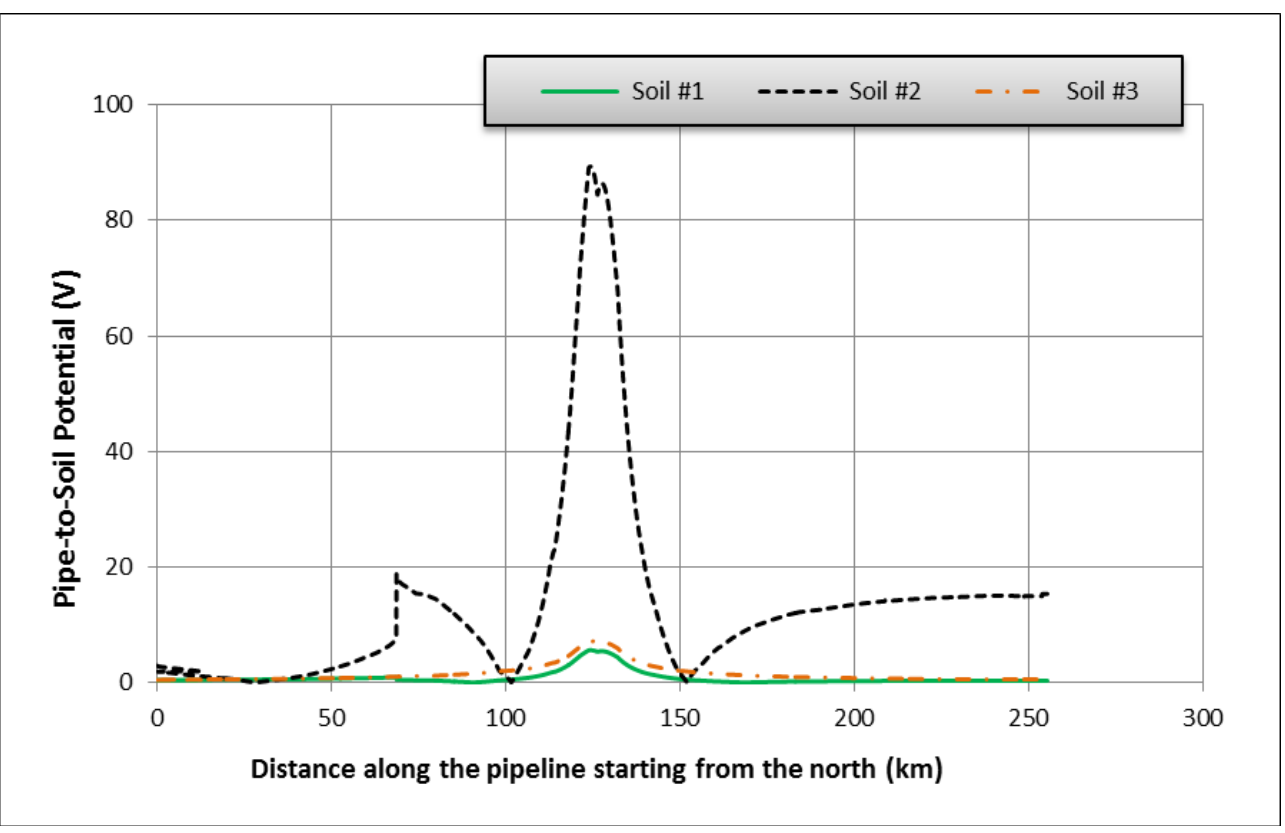

Figure 9. Pipe-to-soil potentials in different soil structures. 


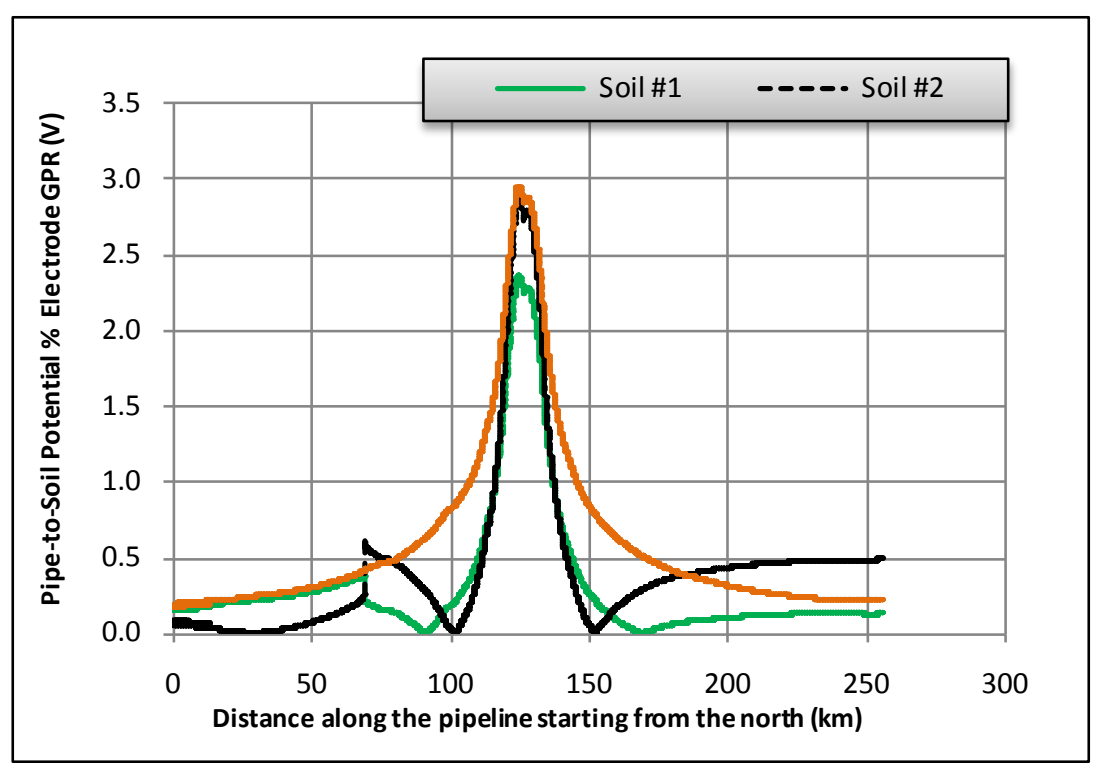

Figure 10. Pipe-to-soil potentials as a percentage of HVDC electrode GPR in different soil structures.

Figure 9 shows clearly that soil structure characteristics have a significant impact on the level of coupling between the HVDC ground and the pipe. It is understandable that the low-over-high resistivity soil type (Soil \#2) will generate the highest conductive coupling level. We also note that the pipe-to-soil potentials in Soil \#1 and Soil \#3 that have the same bottom layer soil resistivity appears to provide similar interference levels, or to be insensitive to the top layer: This is due to the relatively small depth (compared with the overall size of the system), i.e., a $2 \mathrm{~m}$ thickness of the top layer, of the soil resistivity variations. This underscores the need and importance to know soil resistivities to considerable depths to predict the performance of such a system.

Figure 10 reveals an interesting result. Although the actual interference levels vary dramatically with the change of the soil resistivity (compare Soil \#1 and Soil \#2), the coupling factor (ratio of the maximum pipeto-soil potential to HVDC electrode GPR) appears to be much less sensitive to the soil structure. In this case, they all lie between $2 \%$ to $3 \%$. However, it is important to point out the conclusion can be very different if the pipe is much closer to the electrode and the contrast ratio of soil resistivity becomes lager. Indeed, if we consider the limiting case of an almost metallic top layer soil and plastic bottom layer, we would naturally conclude that the HVDC GPR will transfer $100 \%$ to any other location (e.g., the pipeline).

On the other hand, if the bottom layer is metallic and the top layer is very resistive, then the injected HVDC current would naturally travel vertically to the deep soil and will drop rapidly along the horizontal direction.

\section{Conclusions}

Detailed analysis of HVDC ground current effects on neighboring metallic utilities is an absolute requirement. Accurate models of HVDC ground electrodes and buried metallic pipelines in complex soil structures must be built to compute leakage currents, potentials, and evaluate the interference levels for safety assessment and corrosion rates .This paper discussed and demonstrated advanced methods for evaluating and analyzing electromagnetic interference caused by HVDC electrodes. The study that is described in this paper is based on a realistic case and includes comparisons between field measurements and computed results that validate the computation method and computer models that were developed to describe accurately the existing system.

The resulting computations not only describe the interference levels in the vicinity of a ground electrode, but also demonstrate how the soil structure influences the predicted DC interference levels. It is critical to model the system as built in order to provide accurate data to determine the necessary mitigation required to avoid pipeline damage and to ensure personnel safety and the integrity of the equipment.

The effectiveness of various mitigation measures that reduce the DC interference level to acceptable values will be discussed in a future paper. 


\section{References}

[1] BS/EN50162-2004 (2005) Protection against Corrosion by Stray Current from Direct Current Systems. British-Adopted European Standard.

[2] GB/T21447-2008 (2008) Specification for External Corrosion Control for Steel Pipeline. Chinese National Standard.

[3] NACE SP0169-2013 (2013) Control of External Corrosion on Underground or Submerged Metallic Piping Systems. NACE.

[4] Peabody, A.W. and Siegfried, C.G. (1974) Corrosion Control Problems and Personnel Hazard Control Problems Caused by HVDC and HVAC Transmission Systems on Non-Associated Underground Facilities. CIGRE International Conference on Large High Voltage Electric Systems. 